\title{
GC-MS Analysis and Antimicrobial Screening of Essential Oil from Lemongrass (Cymbopogon citratus)
}

\author{
Mahmoud Mohamed Ali, Mohammad Abdi Yusuf, Mohamed N. Abdalaziz* \\ Department of Chemistry, Faculty of Pure and Applied Science, International University of Africa, Khartoum, Sudan
}

\section{Email address:}

mohdnasr19@gmail.com (M. N. Abdalaziz)

*Corresponding author

\section{To cite this article:}

Mahmoud Mohamed Ali, Mohammad Abdi Yusuf, Mohamed N. Abdalaziz. GC-MS Analysis and Antimicrobial Screening of Essential Oil from Lemongrass (Cymbopogon citratus). International Journal of Pharmacy and Chemistry. Vol. 3, No. 6, 2017, pp. 72-76. doi: $10.11648 /$ j.ijpc.20170306.11

Received: September 29, 2017; Accepted: October 17, 2017; Published: November 1, 2017

\begin{abstract}
Medicinal plants are source of a great economic value. Plant herbs are naturally gifted at the synthesis of medicinal compounds. The extraction and characterization of bioactive compounds from medicinal plants have resulted in the discovery of new drugs with high therapeutic value. Lemon grass was collected from administration gardens near Burjifartih west Khartoum, and was extracted for its oil by the hydro-distillation method approximately four hours. the extracted oil was analyzed by GC-MS and chemical constituents in the oil were identified, the major compounds in the oil were, citral (34.8\%), neral (30.72\%), $\beta$-myrecene (11.28\%), geraniol (5.54\%), 1,3,4-trimethyl 3-cyclohexene-1-Carboxaldehyde (2.20\%), citonellol (1.34\%). The compounds, D-Limonene (0.03\%), Geranyl Acetate (0.57\%) and Bicyclo [3.1.1] heptane-2-Carboxaldehyde-6,6dimethyl $(0.23 \%)$ were the minor compounds. antibacterial and antifungal activities of the oil were tested, all tested microorganisms were sensitive and no resistant was not observed, all growth inhibition zones occurred in the high activity level which is $(>18 \mathrm{~mm})$.
\end{abstract}

Keywords: Extraction, Lemon Grass, GC-MS, Antimicrobial

\section{Introduction}

Essential oils (EOs) are very interesting natural plant products and among other qualities they possess various biological properties. The term "biological" comprises all activities that these mixtures of volatile compounds (mainly mono- and sesquiterpenoids, benzenoids, phenylpropanoids, etc.) exert on humans, animals, and other plants. Natural essential oils are volatile, fragrant and pleasant tasting oils obtained from leaves, roots, flowers, seeds and fruits. EOs are low-volume high-value products used in perfumery, cosmetics, feed, food, beverages, and pharmaceutical industries. Essential oils have been in use in both religion and medicine for thousands of years.

The power of living products (herbals and essential oils) lies in the combination of their elements, and the trace components are every bit as important as their main constituents. In fact, it seems to be that the minor constituents have a synergistic (controlling and strengthening) effect on the main constituents. Many of these trace elements enable the herbal or oil to heal more efficiently and without the nasty side effects experienced when using the synthetic reconstructions (drugs or oils) that do not contain the trace elements. The modern world is bringing aromatherapy into building designs and medical practices for this purpose.

Medicinal plants are source of a great economic value. Plant herbs are naturally gifted at the synthesis of medicinal compounds. The extraction and characterization of bioactive compounds from medicinal plants have resulted in the discovery of new drugs with high therapeutic value. Treatment using medicines of natural origin is gaining momentum nowadays on account of increasing concern about potentially harmful synthetic additives [1]. Cymbopogan citratus (commonlynamed, lemongrass), is a great interest due to its commercially valuable essential oils and widely used in food technology as well as in traditional medicine.

Owing to the new attraction for natural products like essential oils, despite their wide use and being familiar to us 
as fragrances, it is important to develop a better understanding of their mode of biological action for new applications in human health, agriculture and the environment.

People nowadays are more aware on health issue due to the emergence of new diseases. Treatment using plant-based medicine appears to be an alternative approach due to the adverse effects associated with the use of synthetic drugs. The genetic diversity of plants has provided us not only survival, but a high degree of comfort and the most important thing of all is the potential treatment for various diseases [2]. The anti-oxidant properties of essential oils might be encouraging to consider them as natural oxidant in nutraceuticals and pharmaceutical preparations. In recent years, there is an increasing interest in finding antioxidant photochemical, because they can inhibit the propagation of free radical reactions, protect the human body from diseases [3]; [4]; [1] and retard lipid oxidative rancidity in foods [5].

One of the common chronic disease infected many people nowadays is diabetes mellitus which is a metabolic disorder affecting carbohydrate, fat and protein metabolism. The worldwide survey reported that the percentage of people being affected by this disease is increasing yearly. The increase in demand in industrially developed countries to use alternative approaches to treat diabetes, such as plant based medicines, is due to the side effects associated with the use of insulin and oral hypoglycemic agents [6]. Therefore, it is crucial to search for newer anti-diabetic agents that possess less side effects and safe to be used on human bodies.

Gout is another common chronic disease nowadays which occurs in individuals who have high serum uric acid levels. The treatment of gout entails the use of therapeutic agents such as xanthine oxidase inhibitors (XOI). The prototypical of allopurinol as XOI has been the cornerstone of the clinical management of gout and conditions associated with hyperuricemia for several decades [7]. However, it is important to note that the current XOI, including allopurinol possess some side effects where some people develop rash as they are allergic to allopurinol [8]

Furthermore, severe reactions also occur including liver function abnormalities [7].

The most apprehension complication with regard to allopurinol is called "allopurinol hypersensitivity syndrome" which can sometimes be fatal and life threatening [8]; [9].

Hence, introducing natural remedy as new source of gout medication is highly granted. There are studies aimed to find the potentiality of lemongrass essential oil which could be the alternative approach for the treatment of chronic diseases such as diabetes, gout and other related diseases to substitute the conventional drugs which possesses some adverse effects.

\section{Material and Methods}

Plant material

Lemon grass was collected at administration gardens near BurjiFatih in Khartoum city Sudan in 2016. The leaves of lemon grass were cut into small $10 \mathrm{~cm}$ long pieces and crushed as possible, weighed using aluminum foil and transferred into 5 liter round- bottomed flask to which was added $200 \mathrm{ml}$ of distilled water, and distilled for four hours using a Clevenger apparatus (AOAC,1990) [10]. The total distillation time was approximately (including about one hour for the oil to start distilling). Since the essential oil of lemon grass is less dense than water, the oil floated on the top of the water. The oil was separated from the water using a syringe. Anhydrous sodium sulfate was used to dry the oil.

Analysis of Lemon Grass Oil

Lemon grass oil was analyzed in a Shimadzu Gas Chromatograph (Model GC.MS-QP2010 Ultra) coupled with a non-polar Rtx-MS capillary column 30meter in length, diameter $(0.25 \mathrm{~mm})$ and thickness $(0.25 \mu \mathrm{l})$ using a mass spectrometer detector and helium was used as a mobile phase (carrier gas). The instrument was connected to a computer coupled with special software that was used to analyze the data.

Retention indices (RI), using n-alkanes were used as the basis. The percent of each compound was based on the peak area divided by the total area of component peaks. The temperature range was from $50-300^{\circ} \mathrm{C}$, with a temperature program rate of $10^{\circ} \mathrm{C} / \mathrm{min}$, starting at three minutes and finishing at thirty minutes. The pressure applied in this experiment was $100 \mathrm{kpa}$ with a total flow of $50 \mathrm{ml} / \mathrm{min}$ and $1.69 \mathrm{ml} / \mathrm{min}$ of column flow. The injection, ion source and the interface temperatures were $300^{\circ} \mathrm{C}, 200^{\circ} \mathrm{C}$ and $250^{\circ} \mathrm{C}$ respectively.

Testing of Extracted oil for Antibacterial Activity on standard bacteria

The cup-plate agar diffusion method was adopted with small modifications according to [12]. To assess the antibacterial activity of the prepared extract. $0.01 \mathrm{ml}$ of each standardized bacterial stock suspension colony- forming units per $\mathrm{ml}$ had been thoroughly mixed with $20 \mathrm{ml}$ of sterile nutrient agar. $20 \mathrm{ml}$ of the inoculated nutrient agar will be distributed into sterile Petri dishes. The agars plate will be left to set and in each of these plates 1 cup, $10 \mathrm{~mm}$ in diameter, was cut using a sterile cork borer No. 4 and the agar discs removed. The cup had been filled with $0.1 \mathrm{ml}$ of the extract using micro titer-pipette and allowed to diffuse at room temperature for two hours. The plates were incubated in the upright position at $37^{\circ} \mathrm{C}$ for 18 hours. Methanol was used as a dissolving chemical and had been carried out as control. After incubation the diameters of the results and growth inhibition zones were measured and tabulated. Bacteria tested in this way were four: (E. coli, Staphylococcus aureus, Salmonella typhi, Klebsellapneuemoniae.).

Testing for anti-fungal activity

The same method of the bacteria was adopted. Instead of nutrient agar, Sabouraud-dextrose agar had been used. The inoculated media were incubated at $25^{\circ} \mathrm{C}$ for one day for the Candida albicans and Aspergillusniger. 


\section{Results}

Table 1. Major compositions of lemongrass (cymbopogon citratus) oil obtained by GC/MS analysis.

\begin{tabular}{ll}
\hline Compound Name & Relative peak area\% \\
\hline$\beta$ Myrcene & 11.28 \\
Geraniol & 5.54 \\
D-Limonene & 0.03 \\
Citral & 34.80 \\
Citronellol & 1.34 \\
Geranyl Acetate & 0.57 \\
Neral & 30.72 \\
1,3,4-trimethyl 3-cyclohexene-1 Carboxaldehyde & 2.20 \\
\hline
\end{tabular}

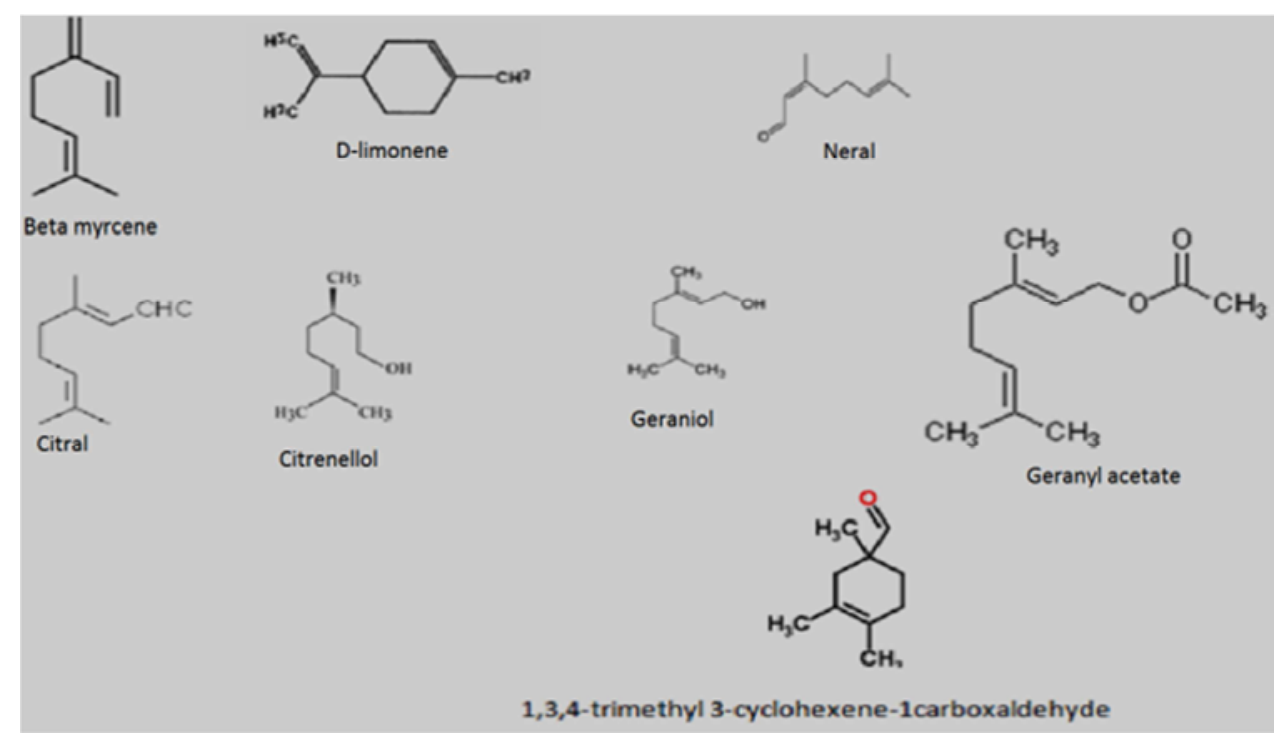

Figure 1. Structures of the major compounds that were obtained by GC-MS analysis.

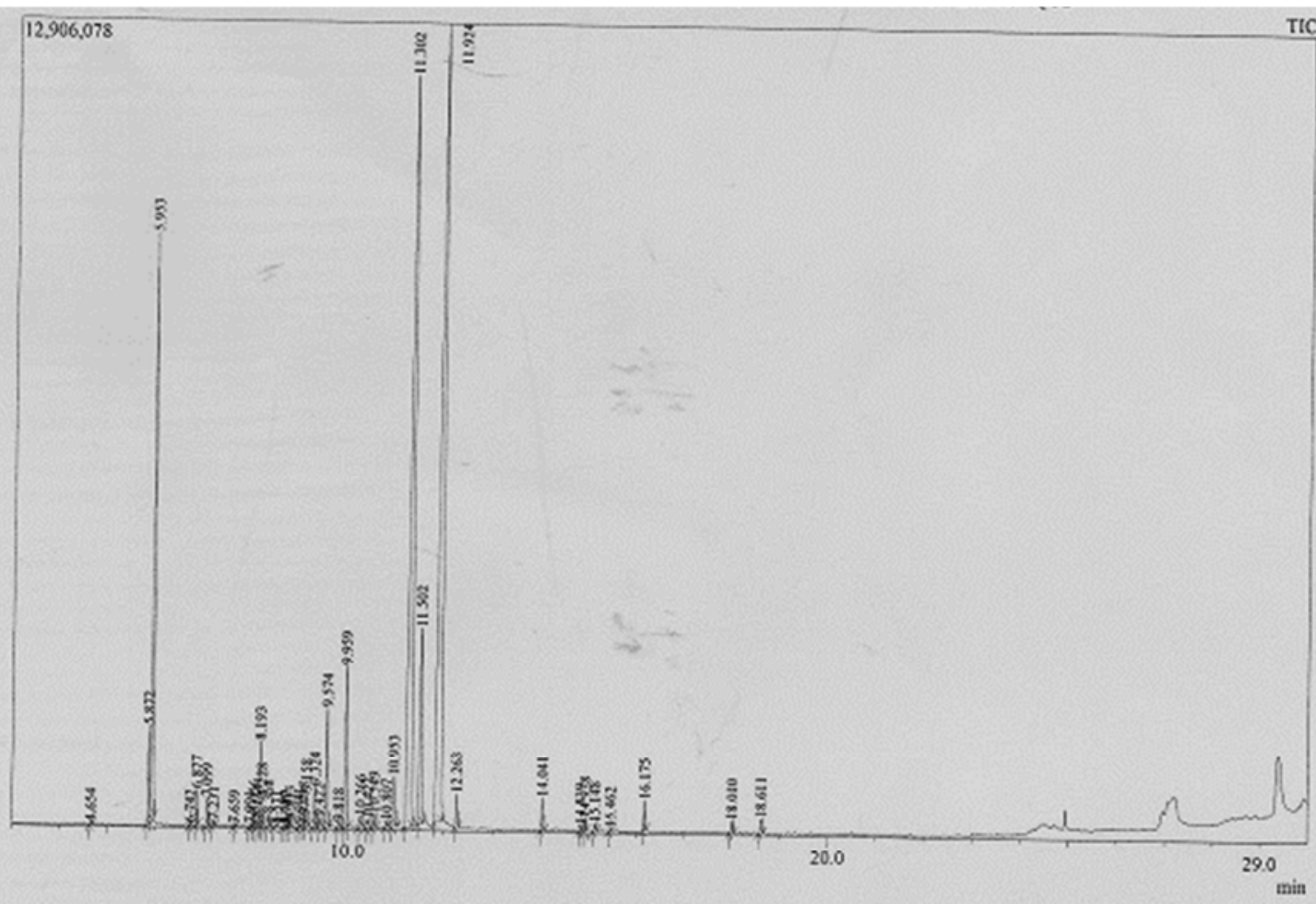

Figure 2. Chromatogram of lemon grass oil obtained from GC/MS. 
Table 2. Shows antibacterial and antifungal activity obtained by the microbiology tests.

\begin{tabular}{lll}
\hline Bacteria/fungi & $\begin{array}{l}\text { Lemongrass oil growth } \\
\text { inhibition zones in mm }\end{array}$ & activity \\
\hline E. coli & 23 & +++ \\
Staphylococcus aureus & 38 & +++ \\
Salmonella tiphi & 23 & +++ \\
Klebsellapneuemoniae & 30 & +++ \\
Aspergillusniger & 19 & +++ \\
Candida alpicans & 29 & +++ \\
\hline
\end{tabular}

+++ means high activity.

The methanol control had not shown any inhibition zone meaning that it has no activity against microorganisms.

\section{Discussion}

GC-MS analyses revealed the presence of 43 compounds but 8 compounds were selected as the compounds that were expected their presence in essential oil. It is well-known that grass plants produce terpenoidal hydrocarbons and EOs that can be grouped as medicinal, industrial, and perfumery, depending on their chemical composition.

The major components were citral (34.8\%), neral (30.72\%), $\beta$-myrcene (11.28\%), geraniol (5.54\%)1,3,4-trimethyl -3cyclohexene-1-carboxaldehyde (2.20\%), and citronellol (1.34\%). Geranyl acetate (0.57\%), Bicyclo[3.1.1] heptane-2Carboxaldehyde-6,6-dimethyl(0.23\%) and D-lemonene $(0.03 \%)$ were minor but significant components (Table 1$)$. The quality of lemon grass is generally determined by its citral content. Citral (3,7- dimethyl-2,6-octadienal) consists of the cis-isomer geranial and the trans-isomer neral. These two oxygenated monoterpenes are present in our LGEO of about $65.52 \%$ Also, LGEO had a high content of oxygenated monoterpenes and low amounts of monoterpene hydrocarbons, sesquiterpene hydrocarbons, and oxygenated sesquiterpenes. Therefore, LGEO extracted by steam distillation may be classified as a 'Citral Chemo type. Comparing the chemical constituents of this LGEO with the compositions of that of Indian oils, with approximately the same content of neral and geranial, whereas the African oils contain high levels of myrcene. Plant clones especially rich in citral have been developed for the cosmetics and chemical industries. According to [11], there are two types or clones of this aromatic herb species on the basis of the relative composition of the EO the East Indian type with high amount of myrcene (38\%) and low citral (7\%), and the West Indian type with little or no myrcene $\left(0 \_12 \%\right)$ and a high citral content (up to $86 \%$ ). Such differences in the chemical composition have been attributed to a range of factors, including geographical location, climatic conditions, time of harvest, age of the plant, and the method of distillation.

Antibacterial and antifungal activity, four bacteria and two fungi were tested our essential oil to know its activity against microbes, and the all tested microbes were sensitive, a clear zone of growth inhibition $(>18 \mathrm{~mm})$ was evident.

\section{Conclusion}

Our findings indicate that lemon grass contains high contents of citral and its cis-isomer (neral), beta myrecene and other aldehydic compounds, it also contained mono terpenes esquiterpens which are included among them oxygenated and non- oxygenated compounds as well as it contained phenolic compounds. The extracted lemongrass essential oil indicated a high sensitivity of bactericidal and fungicidal, all tested microorganisms their growth was inhibited by lemon grass extracted essential oil, this show us that lemon grass oil is a potentially valuable antifungal and anti-inflammatory agent for the prevention and treatment of acute inflammatory skin conditions. Furthermore, there is growing evidence that LGEO in vapor phase is an effective antifungal system that has several advantages over the liquid phase, such as greater potency so that lower doses are required for the same effect. The exact mechanism of the anti-inflammatory effect of the LGEO is unclear the present study demonstrates the need for more investigations to identify the active chemical constituents of LGEO responsible for its therapeutic properties as well as their specific mechanism of action.

\section{References}

[1] Reische, D. L. 1998. Antioxidant in food lipids. In i. C. (Eds.), Chemistry, Nutrition and Biotechnology, New York: Marcel Dekker, p423-448.

[2] Payne, G., Bringi, V., Prince, C. and Shuler, M. 1991. PlantCell and Tissue Culture in Liquid Systems. The Questfor Commercial Production of Chemicals from PlantCell Culture: 3. New York: Oxford University Press.

[3] Kinsella, J. E. 1993. Possible mechanisms for the protective role of antioxidants in wine and plant foods. Food Technology: 85-89.

[4] Terao, J. and Piskula, M. K. 1997. Flavonoids as inhibitors, The Journal of Physiology 555: 589-606.

[5] Duthie, G. G. 1993. Lipid Peroxidation. European Journal of Clinical Nutrition 47(11):759-764.

[6] Marles, R. and Farnsworth, N. 1994. Plants as sources of antidiabetic agents. In: Wagner, H.,Farnsworth, N. R.

[7] Fields, M., Lewis, C. G. and Lure, M. D. 1996. Allopurinol, an Inhibitor of Xanthine Oxidase, Reduces Uric Acid Levels and Modifies the Signs Associated with Copper Deficiency in Rats Fed Fructose. Free Radical Biologyand Medicine 20 (4): $595-600$.

[8] Kong L. D., Zhang Y., Pan X., Tan R. X. and ChengC. H. K. 2000. Inhibition of Xanthine Oxidase by Liquiritigenin and Isoliquiritigenin Isolated from Sino franch etiachinensi. Cellular and Molecular LifeSciences 57: 500-505.

[9] Berry, C. E. and Hare, J. M. 2004. Xanthine Oxidoreductase and Cardiovascular Disease: The MolecularMechanisms and Pathophysiological Implications. The Journal of Physiology 555: 589-606. 
[10] Association of analytical chemistry(AOAC), 1990. Official methods of analysis of the association of official analytical chemists, 15 th edition, page 951-952.

[11] Akhila A.2010. Essential oil-bearing grasses: the genus Cymbopogon.
[12] Kavanagh, F. (1972). Analytical microbiology. Vol. 11. Academic press (pub) New York and London. Pp 11. 